\title{
On the non-existence of simple congruences for quotients of Eisenstein series
}

by

Michael Dewar (Kingston, Ont.)

1. Introduction. Define $p(n)$ to be the number of ways of writing $n$ as a sum of non-increasing positive integers. Ramanujan famously established the congruences

$$
\begin{aligned}
p(5 n+4) & \equiv 0 \bmod 5, \\
p(7 n+5) & \equiv 0 \bmod 7, \\
p(11 n+6) & \equiv 0 \bmod 11,
\end{aligned}
$$

and noted that there does not appear to be any other prime for which the partition function has equally simple congruences. Ahlgren and Boylan [1] build on the work of Kiming and Olsson [5] to prove that there truly are no other such primes. For large enough primes $l$, Sinick [7] and the author [3] prove the non-existence of simple congruences

$$
a(\ln +c) \equiv 0 \bmod l
$$

for wide classes of functions $a(n)$ related to the coefficients of modular forms. However, all of the modular forms studied in [1], [7] and [3] are non-vanishing on the upper half-plane. Here we prove the non-existence of simple congruences (when $l$ is large enough) for ratios of Eisenstein series.

Let $\sigma_{m}(n):=\sum_{d \mid n} d^{m}$ and define the Bernoulli numbers $B_{k}$ by $t /\left(e^{t}-1\right)$ $=\sum_{k=0}^{\infty} B_{k} t^{k} / k$ !. For even $k \geq 2$, set

$$
E_{k}(\tau):=1-\frac{2 k}{B_{k}} \sum_{n=1}^{\infty} \sigma_{k-1}(n) q^{n} .
$$

Note that $E_{2} \equiv E_{4} \equiv E_{6} \equiv 1$ modulo 2 and 3. Berndt and Yee [2] prove congruences for the quotients of Eisenstein series in Table 1, where $F(q):=$ $\sum a(n) q^{n}$. An obviously necessary requirement for the congruences in the $n \equiv 2 \bmod 3$ column of Table 1 is that there are simple congruences of the

2010 Mathematics Subject Classification: Primary 11F33; Secondary 11F30, 11F11.

Key words and phrases: Ramanujan congruences, Eisenstein series, Tate cycle. 
Table 1. Congruences of Berndt and Yee [2]

\begin{tabular}{ccc}
\hline$F(q)$ & $n \equiv 2 \bmod 3$ & $n \equiv 4 \bmod 8$ \\
\hline $1 / E_{2}$ & $a(n) \equiv 0 \bmod 3^{4}$ & \\
$1 / E_{4}$ & $a(n) \equiv 0 \bmod 3^{2}$ & \\
$1 / E_{6}$ & $a(n) \equiv 0 \bmod 3^{3}$ & $a(n) \equiv 0 \bmod 7^{2}$ \\
$E_{2} / E_{4}$ & $a(n) \equiv 0 \bmod 3^{3}$ & \\
$E_{2} / E_{6}$ & $a(n) \equiv 0 \bmod 3^{2}$ & $a(n) \equiv 0 \bmod 7^{2}$ \\
$E_{4} / E_{6}$ & $a(n) \equiv 0 \bmod 3^{3}$ & \\
$E_{2}^{2} / E_{6}$ & $a(n) \equiv 0 \bmod 3^{5}$ & \\
\hline
\end{tabular}

form $a(3 n+2) \equiv 0 \bmod 3$. All but the first form in Table 1 are covered by the following theorem.

Theorem 1.1. Let $r \geq 0$ and $s, t \in \mathbb{Z}$. If $E_{2}^{r} E_{4}^{s} E_{6}^{t}=\sum a(n) q^{n}$ has a simple congruence $a(\ln +c) \equiv 0 \bmod l$ for the prime $l$, then either $l \leq$ $2 r+8|s|+12|t|+21$ or $r=s=t=0$.

This theorem gives an explicit upper bound on primes $l$ for which there can be congruences of the form $a(\ln +c) \equiv 0 \bmod l^{k}$ as in the middle column of Table 1 .

REMARK 1.2. See Remark 4.1 for a slight improvement of Theorem 1.1 in some cases.

EXAMPLE 1.3. The form $E_{6} / E_{4}^{12}$ can only have simple congruences for $l \leq 129$. Of these, the primes $l=2$ and 3 are trivial with $E_{4} \equiv E_{6} \equiv 1 \bmod l$. For the remaining primes, the only congruences are

$$
a(\ln +c) \equiv 0 \bmod 17, \quad \text { where }\left(\frac{c}{17}\right)=-1 .
$$

Mahlburg [6] shows that for each of the forms in Table 1 except $1 / E_{2}$, there are infinitely many primes $l$ such that for any $i \geq 1$, the set of $n$ with $a(n) \equiv 0 \bmod l^{i}$ has arithmetic density 1 . On the other hand, our result shows that (for large enough $l$ ) every arithmetic progression modulo $l$ has at least one non-vanishing coefficient modulo $l$.

Section 2 recalls certain definitions and tools from the theory of modular forms. Simple congruences are reinterpreted in terms of Tate cycles, which are reviewed in Section 3. Section 4 proves Theorem 1.1.

2. Preliminaries. A modular form of weight $k \in \mathbb{Z}$ on $\mathrm{SL}_{2}(\mathbb{Z})$ is a holomorphic function $f: \mathbb{H} \rightarrow \mathbb{C}$ which satisfies

$$
f\left(\frac{a \tau+b}{c \tau+d}\right)=(c \tau+d)^{k} f(\tau)
$$


for every $\left(\begin{array}{ll}a & b \\ c & d\end{array}\right) \in \mathrm{SL}_{2}(\mathbb{Z})$, and which is holomorphic at infinity. Modular forms have Fourier expansions in powers of $q=e^{2 \pi i \tau}$. For any prime $l \geq 5$, let $\mathbb{Z}_{(l)}=\{a / b \in \mathbb{Q}: l \nmid b\}$. We denote by $M_{k}$ the set of all weight $k$ modular forms on $\mathrm{SL}_{2}(\mathbb{Z})$ with $l$-integral Fourier coefficients. Although $E_{k}$ is a modular form of weight $k$ whenever $k \geq 4, E_{2}$ is called a quasi-modular form since it satisfies the slightly different transformation rule

$$
E_{2}\left(\frac{a \tau+b}{c \tau+d}\right)=(c \tau+d)^{2} E_{2}(\tau)-\frac{6 i c}{\pi}(c \tau+d) .
$$

Definition 2.1. If $l$ is a prime, then a Laurent series $f=\sum_{n \geq N} a(n) q^{n}$ $\in \mathbb{Z}_{(l)}((q))$ has a simple congruence at $c \bmod l$ if $a(l n+c) \equiv 0 \bmod l$ for all $n$.

Lemma 2.2. Suppose that $l$ is prime and that $f=\sum a(n) q^{n}$ and $g=$ $\sum b(n) q^{n} \in \mathbb{Z}_{(l)}((q))$ with $g \not \equiv 0 \bmod l$. The series $f$ has a simple congruence at $c \bmod l$ if and only if the series $\mathrm{fg}^{l}$ has a simple congruence at $c \bmod l$.

Proof. It suffices to consider the reductions $\bmod l$ of the series

$$
\left(\sum a(n) q^{n}\right)\left(\sum b(n) q^{l n}\right) \equiv \sum_{n}\left(\sum_{m} b(m) a(n-l m)\right) q^{n} \bmod l .
$$

If $a(n)$ vanishes when $n \equiv c \bmod l$, then the inner sum on the right hand side will also vanish for $n \equiv c \bmod l$. The converse follows via multiplication by $\left(\sum b(n) q^{n}\right)^{-l}$ and repetition of this argument.

Our main tool is Ramanujan's $\Theta$ operator

$$
\Theta:=\frac{1}{2 \pi i} \frac{d}{d \tau}=q \frac{d}{d q} .
$$

For any prime $l$ and any Laurent series $f=\sum a(n) q^{n} \in \mathbb{Z}_{(l)}((q))$, by Fermat's Little Theorem

$$
\Theta^{l} f=\sum a(n) n^{l} q^{n} \equiv \sum a(n) n q^{n}=\Theta f \bmod l .
$$

We call the sequence $\Theta f, \ldots, \Theta^{l} f \bmod l$ the Tate cycle of $f$. Note that $\Theta^{l-1} f \equiv f \bmod l$ is equivalent to $f$ having a simple congruence at $0 \bmod l$.

We now recall some facts about the reductions of modular forms mod $l$. See Swinnerton-Dyer [8, Section 3] for the details on this paragraph. There are polynomials $A(Q, R), B(Q, R) \in \mathbb{Z}_{(l)}[Q, R]$ such that

$$
A\left(E_{4}, E_{6}\right)=E_{l-1}, \quad B\left(E_{4}, E_{6}\right)=E_{l+1} .
$$

Reduce the coefficients of these polynomials modulo $l$ to get $\tilde{A}, \tilde{B} \in \mathbb{F}_{l}[Q, R]$. Then the polynomial $\tilde{A}$ has no repeated factor and is prime to $\tilde{B}$. Furthermore, the $\mathbb{F}_{l}$-algebra of reduced modular forms is naturally isomorphic to

$$
\frac{\mathbb{F}_{l}[Q, R]}{\tilde{A}-1}
$$


via $Q \rightarrow E_{4}$ and $R \rightarrow E_{6}$. Whenever a power series $f$ is congruent to a modular form, define the filtration of $f$ by

$$
\omega(f):=\inf \left\{k: f \equiv g \in M_{k} \bmod l\right\} .
$$

If $f \in M_{k}$, then for some $g \in M_{k+l+1}, \Theta f \equiv g \bmod l$. The next lemma also follows from [8, Section 3].

Lemma 2.3. Let $l \geq 5$ be prime, $f \in M_{k_{1}}, f \not \equiv 0 \bmod l$ and $g \in M_{k_{2}}$.

(1) If $f \equiv g \bmod l$, then $k_{1} \equiv k_{2} \bmod l-1$.

(2) $\omega(\Theta f) \leq \omega(f)+l+1$ with equality if and only if $\omega(f) \not \equiv 0 \bmod l$.

(3) If $\omega(f) \equiv 0 \bmod l$, then for some $s \geq 1, \omega(\Theta f)=\omega(f)+(l+1)-$ $s(l-1)$.

(4) $\omega\left(f^{i}\right)=i \omega(f)$.

The natural grading induced by (2.1) provides a key step in the following lemma which is taken from the proof of [5, Proposition 2].

LEMma 2.4. A form $f \in M_{k}$ with $\Theta f \not \equiv 0 \bmod l$ has a simple congruence at $c \not \equiv 0 \bmod l$ if and only if $\Theta^{(l+1) / 2} f \equiv-\left(\frac{c}{l}\right) \Theta f \bmod l$.

Proof. Since $\Theta$ satisfies the product rule, we have

$$
\begin{aligned}
\Theta^{l-1}\left(q^{-c} f\right) & \equiv \sum_{i=0}^{l-1}\left(\begin{array}{c}
l-1 \\
i
\end{array}\right)(-c)^{l-1-i} q^{-c} \Theta^{i} f \bmod l \equiv \sum_{i=0}^{l-1} c^{l-1-i} q^{-c} \Theta^{i} f \bmod l \\
& \equiv c^{l-1} q^{-c} f+\sum_{i=1}^{l-1} c^{l-1-i} q^{-c} \Theta^{i} f \bmod l .
\end{aligned}
$$

A simple congruence for $f$ at $c \not \equiv 0 \bmod l$ is equivalent to a simple congruence for $q^{-c} f$ at $0 \bmod l$, which in turn is equivalent to $\Theta^{l-1}\left(q^{-c} f\right) \equiv$ $q^{-c} f \bmod l$. This is equivalent to $0 \equiv \sum_{i=1}^{l-1} c^{l-1-i} q^{-c} \Theta^{i} f \bmod l$, by the computation above, and hence to $0 \equiv \sum_{i=1}^{l-1} c^{l-1-i} \Theta^{i} f \bmod l$. By Lemma 2.3 (2) and $(3)$, for $1 \leq i \leq(l-1) / 2$ we have

$$
\omega\left(\Theta^{i} f\right) \equiv \omega\left(\Theta^{i+(l-1) / 2} f\right) \equiv \omega(f)+2 i \bmod l-1 .
$$

By Lemma 2.3(1) and the natural grading (filtration modulo $l-1$ ), the only way for the given sum to be zero is if for all $1 \leq i \leq(l-1) / 2$ we have

$$
c^{l-1-i} \Theta^{i} f+c^{l-1-(i+(l-1) / 2)} \Theta^{i+(l-1) / 2} f \equiv 0 \bmod l,
$$

which happens if and only if

$$
\Theta^{i+(l-1) / 2} f \equiv-c^{(l-1) / 2} \Theta^{i} f \equiv-\left(\frac{c}{l}\right) \Theta^{i} f \bmod l,
$$

which happens if and only if

$$
\Theta^{(l+1) / 2} f \equiv-\left(\frac{c}{l}\right) \Theta f \bmod l .
$$


Lemma 2.5. Let $a, b, c \geq 0$ be integers and let $l>11$ be prime. Then $\omega\left(E_{l+1}^{a} E_{4}^{b} E_{6}^{c}\right)=a l+a+4 b+6 c$.

Proof. Since $E_{l+1}^{a} E_{4}^{b} E_{6}^{c} \in M_{a l+a+4 b+6 c}$, it suffices to show that $\tilde{A}(Q, R)$ does not divide $\tilde{B}(Q, R)^{a} Q^{b} R^{c}$. However $\tilde{A}$ has no repeated factors and is prime to $\tilde{B}$ and so it suffices to show that $\tilde{A}$ does not divide $Q R$. But $Q R$ has weight 10 and $E_{l-1}$ has weight $l-1>10$ so this is impossible.

3. The structure of Tate cycles. The framework we use below follows Jochnowitz [4]. Let $f \in M_{k}$ be such that $\Theta f \not \equiv 0 \bmod l$. Recall from Section 2 that the Tate cycle of $f$ is the sequence $\Theta f, \ldots, \Theta^{l-1} f \bmod l$. With $s \geq 1$ as in (3) of Lemma 2.3, we have

$$
\omega\left(\Theta^{i+1} f\right) \equiv \begin{cases}\omega\left(\Theta^{i} f\right)+1 \bmod l & \text { if } \omega\left(\Theta^{i} f\right) \not \equiv 0 \bmod l \\ s+1 \bmod l & \text { if } \omega\left(\Theta^{i} f\right) \equiv 0 \bmod l .\end{cases}
$$

In particular, when $\omega\left(\Theta^{i} f\right) \equiv 0 \bmod l$, the quantity $s$ which determines the change in filtration also controls the time until the next occurrence of $\omega\left(\Theta^{i} f\right) \equiv 0 \bmod l$. We say that $\Theta^{i} f$ is a high point of the Tate cycle and $\Theta^{i+1} f$ is a low point of the Tate cycle whenever $\omega\left(\Theta^{i} f\right) \equiv 0 \bmod l$. Elementary considerations (see, for example, [4, Section 7] or [3, Section 3]) yield

Lemma 3.1. Let $f \in M_{k}$ with $\Theta f \not \equiv 0 \bmod l$.

(1) If the Tate cycle has only one low point, then the low point has filtration $2 \bmod l$.

(2) The Tate cycle has one or two low points.

Lemma 3.2. Suppose $f \in M_{k}$ has a simple congruence at $c \not \equiv 0 \bmod l$, where $l \geq 5$ is prime, and $\Theta f \not \equiv 0 \bmod l$. Then the Tate cycle of $f$ has two low points. Furthermore, if $\Theta^{i} f$ is a high point, then

$$
\omega\left(\Theta^{i+1} f\right)=\omega\left(\Theta^{i} f\right)+(l+1)-\left(\frac{l+1}{2}\right)(l-1) \equiv \frac{l+3}{2} \bmod l .
$$

Proof. By Lemma 2.4. $\omega(\Theta f)=\omega\left(\Theta^{(l+1) / 2} f\right)$. Hence, the filtration is not monotonically increasing between $\Theta f$ and $\Theta^{(l+1) / 2} f$, so there must be a fall in filtration (and hence a low point) somewhere in the first half of the Tate cycle. We also have $\omega\left(\Theta^{(l+1) / 2} f\right)=\omega(\Theta f)=\omega\left(\Theta^{l} f\right)$ and so by the same reasoning there must be a low point somewhere in the second half of the Tate cycle. By Lemma 3.1, there are exactly two low points in the Tate cycle. Lemma 2.3(2) and (3) give

$$
\omega(\Theta f)=\omega\left(\Theta^{(l+1) / 2} f\right)=\omega(\Theta f)+\left(\frac{l-1}{2}\right)(l+1)-s(l-1)
$$


for some $s \geq 1$. Hence $s=(l+1) / 2$. By the same reasoning, the fall in filtration for the second half of the Tate cycle must also have $s=(l+1) / 2$. The lemma follows.

The proof of Theorem 1.1 uses the above lemma to determine how far the filtration falls, and the bounds of the next lemma to show a corresponding restriction on $l$.

Lemma 3.3. Let $l \geq 5$ be prime and suppose $f \in M_{k}$ has a simple congruence at $c \not \equiv 0 \bmod l$. If $\omega(f)=A l+B$ where $1 \leq B \leq l-1$, then

$$
\frac{l+1}{2} \leq B \leq A+\frac{l+3}{2} .
$$

Proof. Since $B \neq 0, \omega(\Theta f)=(A+1) l+(B+1)$. From the proof of Lemma 3.2 the Tate cycle has a high point before $\Theta^{(l+1) / 2} f$. By Lemma 3.2 . the high point is $\Theta^{i} f$ with $1 \leq i \leq(l-1) / 2$. Hence we have

$$
\omega\left(\Theta^{i} f\right)=A l+B+i(l+1) \equiv B+i \equiv 0 \bmod l .
$$

Together with the restrictions on $B$ and $i$, this congruence implies that $B+i=l$ and $B \geq(l+1) / 2$. Also, by Lemma 2.3 the high point has filtration

$$
\omega\left(\Theta^{l-B} f\right)=\omega(f)+(l-B)(l+1)=(A+l-B+1) l .
$$

Lemma 3.2 implies that the corresponding low point has filtration

$$
\omega\left(\Theta^{l-B+1} f\right)=\left(A-B+\frac{l+3}{2}\right) l+\left(\frac{l+3}{2}\right) .
$$

The fact that $\omega\left(\Theta^{l-B+1} f\right) \geq 0$ implies the second inequality.

If $\Theta f \equiv 0 \bmod l$, then the Tate cycle is trivial and the above lemmas are not applicable. We dispense with this case now.

Lemma 3.4. Let $f=E_{2}^{r} E_{4}^{s} E_{6}^{t}$ where $r \geq 0$ and $s, t \in \mathbb{Z}$. If $l$ is a prime such that $\Theta f \equiv 0 \bmod l$, then either $l \leq 13$ or $r \equiv s \equiv t \equiv 0 \bmod l$.

ExAmple 3.5. We have $\Theta\left(E_{4} E_{6}\right) \equiv 0 \bmod l$ for $l=2,3,11$.

EXAmple 3.6. We have $\Theta\left(E_{2}^{144} E_{4}^{-15} E_{6}^{-14}\right) \equiv 0 \bmod l$ for $l=2,3,5,7,13$.

Note that $\Theta f \equiv 0 \bmod l$ is equivalent to $f$ having simple congruences at all $c \not \equiv 0 \bmod l$.

Proof of Lemma 3.4. Assume $l \geq 17$ and expand $f$ as a power series to get

$$
\begin{aligned}
f=1 & +(-24 r+240 s-504 t) q \\
& +\left(288 r^{2}-5760 r s+12096 r t-360 r+28800 s^{2}\right. \\
& \left.-120960 s t-26640 s+127008 t^{2}-143640 t\right) q^{2}+\cdots .
\end{aligned}
$$


If $\Theta f \equiv 0 \bmod l$, then the coefficients of $q$ and $q^{2}$ vanish modulo $l$. That is,

$$
-24 r+240 s-504 t \equiv 0 \bmod l,
$$

and

$$
\begin{aligned}
& 288 r^{2}-5760 r s+12096 r t-360 r+28800 s^{2} \\
& \quad-120960 s t-26640 s+127008 t^{2}-143640 t \equiv 0 \bmod l .
\end{aligned}
$$

Furthermore, by Lemmas 2.3 (2) and 2.5 and the fact that $E_{2} \equiv E_{l+1} \bmod l$, we have

$$
\omega\left(E_{l+1}^{r} E_{4}^{s} E_{6}^{t}\right) \equiv r+4 s+6 t \equiv 0 \bmod l .
$$

Solving the system of congruences given by (3.3) and (3.1) yields

$$
\begin{aligned}
7 r & \equiv-72 t \bmod l, \\
14 s & \equiv 15 t \bmod l .
\end{aligned}
$$

Substituting (3.4) and (3.5) into 49 times $(3.2)$ yields

$$
-8255520 t \equiv 0 \bmod l \text {. }
$$

Since $8255520=2^{5} \cdot 3^{4} \cdot 5 \cdot 7^{2} \cdot 13$, the lemma follows.

4. Proof of Theorem 1.1. We begin with the trivial observation that $E_{2}^{r} E_{4}^{s} E_{6}^{t}=1+\cdots$ does not have a simple congruence at $0 \bmod l$. Hence, we assume that $E_{2}^{r} E_{4}^{s} E_{6}^{t}$ has a simple congruence at $c \not \equiv 0 \bmod l$, where $l \geq 5$. Since $E_{2} \equiv E_{l+1} \bmod l, E_{l+1}^{r} E_{4}^{s} E_{6}^{t}$ has a simple congruence at $c \bmod l$. Recall that our goal is to show $l \leq 2 r+8|s|+12|t|+21$. Hence, if $l<|s|$ or $l<|t|$ then we are done. Thus we assume $l+s \geq 0$ and $l+t \geq 0$. We also assume $l>11$. Lemma 3.4 allows us to take $\Theta\left(E_{2}^{r} E_{4}^{s} E_{6}^{t}\right) \not \equiv 0 \bmod l$ (otherwise we are done). By Lemma 2.2 we see that

$$
E_{l+1}^{r} E_{4}^{l+s} E_{6}^{l+t} \in M_{(r+10) l+(r+4 s+6 t)}
$$

has a simple congruence at $c \bmod l$. We work with this multiplied form $E_{l+1}^{r} E_{4}^{l+s} E_{6}^{l+t}$ because it is holomorphic (with positive weight) and so our filtration apparatus is applicable. By Lemma 2.5.

$$
\omega\left(E_{l+1}^{r} E_{4}^{l+s} E_{6}^{l+t}\right)=(r+10) l+(r+4 s+6 t) .
$$

We break into four cases depending on the size of $r+4 s+6 t$ :

(1) If $l \leq|r+4 s+6 t|$ then we are done.

(2) If $0<r+4 s+6 t<l$ then by equation (4.1) and the first inequality of Lemma 3.3 , $(l+1) / 2 \leq r+4 s+6 t$ and we are done.

(3) If $r+4 s+6 t=0$, then by Lemma 2.3 .

$$
\omega\left(\Theta E_{l+1}^{r} E_{4}^{l+s} E_{6}^{l+t}\right)=(r+11) l+1-s^{\prime}(l-1)
$$

for some $s^{\prime} \geq 1$. If $l \leq r+13$ then we are done, so it suffices to consider $l>r+13$. Now in order for the filtration above to be non-negative, we must 
have $s^{\prime} \leq r+11$. Now $\omega\left(\Theta E_{l+1}^{r} E_{4}^{l+s} E_{6}^{l+t}\right) \equiv s^{\prime}+1 \bmod l$. By Lemma 2.4 . there must be a high point of the Tate cycle before $\Theta^{(l+1) / 2} E_{l+1}^{r} E_{4}^{l+s} E_{6}^{l+t}$. Let $i$ be the index of the first high point, so $1 \leq i \leq(l-1) / 2$. Then

$$
\omega\left(\Theta^{i} E_{l+1}^{r} E_{4}^{l+s} E_{6}^{l+t}\right) \equiv s^{\prime}+i \equiv 0 \bmod l .
$$

Together with the restrictions on $i$ and $s^{\prime}$ (namely $s^{\prime} \leq r+11<r+13<l$ ), this congruence implies that

$$
s^{\prime} \geq \frac{l+1}{2} .
$$

That is, $l \leq 2 s^{\prime}-1 \leq 2 r+21$ and we are done.

(4) If $-l<r+4 s+6 t<0$, then take $B=l+r+4 s+6 t$ and $A=r+9$. Equation (4.1) and the second inequality of Lemma 3.3 give

$$
l+r+4 s+6 t \leq r+9+\frac{l+3}{2},
$$

which is equivalent to $l \leq 21-8 s-12 t$ and we are done.

REMARK 4.1. Combining these four cases and recalling that the proof assumed $l+s \geq 0, l+t \geq 0$ and $l>11$, we see that if $r+4 s+6 t>0$, then

$$
l \leq \max \{|s|-1,|t|-1,11,2 r+8 s+12 t-1\},
$$

and if $r+4 s+6 t \leq 0$, then

$$
l \leq \max \{|s|-1,|t|-1,11,21-8 s-12 t\} .
$$

Acknowledgements. The author thanks Scott Ahlgren for careful reading of this article and many helpful suggestions. The author also thanks the reviewer for providing constructive comments which clarified the exposition.

\section{References}

[1] S. Ahlgren and M. Boylan, Arithmetic properties of the partition function, Invent. Math. 153 (2003), 487-502.

[2] B. C. Berndt and A. J. Yee, Congruences for the coefficients of quotients of Eisenstein series, Acta Arith. 104 (2002), 297-308.

[3] M. Dewar, Non-existence of Ramanujan congruences in modular forms of level four, Canad. J. Math., to appear.

[4] N. Jochnowitz, A study of the local components of the Hecke algebra mod l, Trans. Amer. Math. Soc. 270 (1982), 253-267.

[5] I. Kiming and J. B. Olsson, Congruences like Ramanujan's for powers of the partition function, Arch. Math. (Basel) 59 (1992), 348-360.

[6] K. Mahlburg, Partition congruences and the Andrews-Garvan-Dyson crank, Proc. Nat. Acad. Sci. U.S.A. 102 (2005), 15373-15376.

[7] J. Sinick, Ramanujan congruences for a class of eta quotients, Int. J. Number Theory, to appear. 
[8] H. P. F. Swinnerton-Dyer, On l-adic representations and congruences for coefficients of modular forms, in: Modular Functions of One Variable, III (Antwerp, 1972), Lecture Notes in Math. 350, Springer, Berlin, 1973, 1-55.

Michael Dewar

Department of Mathematics and Statistics

Queen's University

Kingston, Ontario, K7L 3N6, Canada

E-mail: mdewar@mast.queensu.ca

Received on 13.7.2009

and in revised form on 27.1.2010 